\title{
Exploring Catalysis inside Self-assembled Supramolecular Containers
}

\author{
Lorenzo Cattia, Thomas M. Bräuera, Qi Zhanga, and Konrad Tiefenbacher ${ }^{\star a b}$
}

\begin{abstract}
Our group is interested in applying supramolecular host systems as catalysts for challenging transformations. Although a variety of supramolecular containers has been described in literature, their use as reaction chambers is still underexplored. We herein describe our first steps in this exciting interdisciplinary field of research.
\end{abstract}

Keywords: Catalysis · Cyclization · Host-guest chemistry · Molecular capsule $\cdot$ Supramolecular chemistry

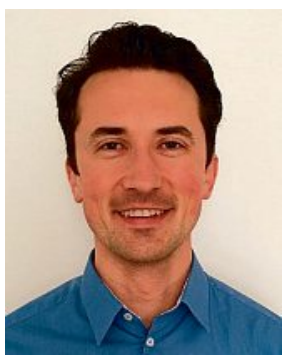

Konrad Tiefenbacher received his chemical basic education at the Technical University of Vienna and the University of Texas in Austin. After finishing his diploma thesis, he pursued his interest in total synthesis of biologically active natural products during a $\mathrm{PhD}$ in the lab of Prof. Mulzer at the University of Vienna. He then moved to Prof. Rebek's lab at The Scripps Research Institute in La Jolla to learn about molecular recognition and selfassembly. In December 2011 he started his independent career as a Juniorprofessor (W1-position) at the Technical University Munich. In June 2016 he was appointed to a dual tenure track assistant professorship at the University of Basel and the ETH Zurich.

\section{Introduction}

Research in supramolecular chemistry has yielded a large variety of container structures capable of encapsulating guest molecules. Early examples involved mainly host structures where the subcomponents were linked with covalent bonds. Typical examples of such covalent containers are the well-studied cyclodextrins and cyclophanes. ${ }^{[1]}$ These molecules feature a hydrophobic interior which is accessible via relatively large openings. On the other side, there are examples of covalent container molecules that do not feature openings large enough for guest exchange and therefore irreversibly entrap molecules present during synthesis ('carcerands').[2] Obviously, such structures are not suitable for catalytic applications which require facile guest exchange. Furthermore, with catalytic applications in mind, the construction of the host structure should be facile and allow the ready preparation of larger amounts of material required for extensive studies. Over the last decades, noncovalently linked host structures have been increasingly investigated where the subcomponents are held together via weak(er) forces: metal-ligand interactions, ${ }^{[3-6]}$ hydrogen bonds ${ }^{[7]}$ or mainly the hydropho- bic effect. ${ }^{[8]}$ In this review, the focus is put on our results concerning catalysis inside a molecular container held together via hydrogen bonds: the hexamer I of resorcin[4]arene (Fig. 1). ${ }^{[9]}$ Readers interested in a broader overview are referred to the respective review articles. ${ }^{[10-17]}$

\section{Reactivity inside Supramolecular Containers}

The reactivity of substrates can be dramatically different inside molecular containers than outside in the regular solution phase. For instance, it was described that pyrophoric white phosphorus is air-stable inside a metal-ligand supramolecular assembly. ${ }^{[18]}$ In another report, orthoesters were cleaved in basic solution inside a self-assembled container, although they are completely stable outside. ${ }^{[19]}$ Why can molecules display a (completely) different reactivity inside supramolecular containers as compared to in the regular solution environment? This has to do with the interactions of the guest molecule and its surrounding host. The host may protect the guest from reactive species like oxygen, which is responsible for the stability of white phosphorous inside a well-fitting
${ }^{\star}$ Correspondence: Prof. Dr. K. Tiefenbacher ${ }^{\text {ab }}$ E-mail: konrad.tiefenbacher@unibas.ch; tkonrad@ethz.ch

aDepartment of Chemistry

University of Basel

St.-Johanns-Ring 19

$\mathrm{CH}-4056$ Basel

bepartment of Biosystems Science and Engineering ETH Zurich

Mattenstrasse 26

$\mathrm{CH}-4058$ Basel

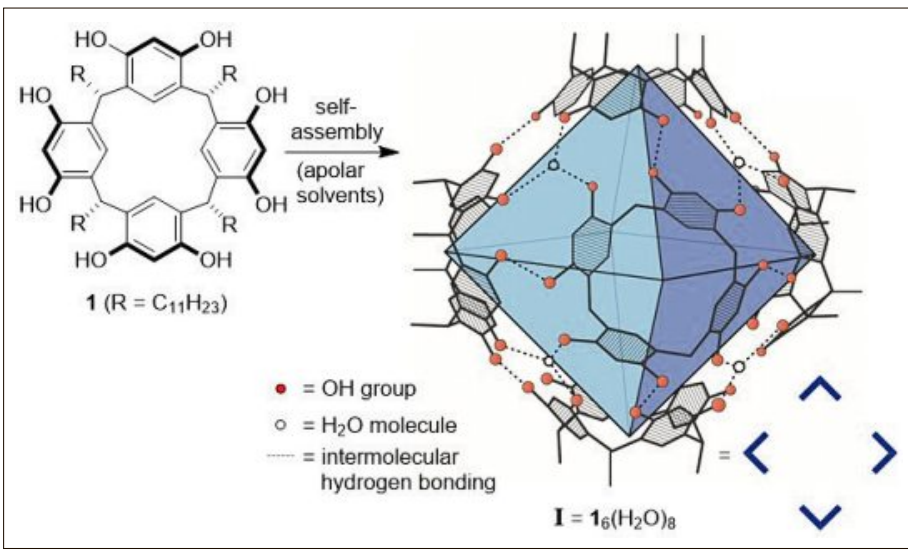

Fig. 1. Hexamer I assembles from six resorcin[4]arene units 1 and eight water molecules. 
container. More suitable for catalytic applications is another aspect of host-guest interaction: stabilization of intermediates and transition states of reactions. Such interactions are responsible for the facile orthoester hydrolysis under basic conditions inside a suitable host system: the aromatic, anionic container stabilizes cationic species inside the cavity due to coulombic and cation- $\pi$ interactions.

Cation- $\pi$ interactions also play an important role in natural enzymes: for instance in the large class of terpene cyclase enzymes, where the aromatic residues of the amino acids phenylalanine, tyrosine and tryptophan are oriented towards cationic charges. ${ }^{[20,21]}$ For an acceleration effect, however, transition states have to be stabilized to a greater extent than intermediates. This is generally believed to be the case since the delocalized cationic charge in the transition state is better stabilized than the localized one on the cationic intermediate.

\subsection{Problem of Product Inhibition}

An important and often encountered roadblock during the development of host structures for catalytic applications is product inhibition. This means that the product binds stronger to the host system than the substrate(s). Especially in bimolecular reactions product inhibition is often encountered: when the host structure binds both substrates in a suitable conformation for the reaction of interest to occur, it will most likely bind the product even stronger due to entropic considerations. Therefore, the host is blocked/inhibited by the product, slowing turnover down or even preventing further conversion completely. How can the problem of product inhibition be resolved? A host has to be designed that binds the transition state better than the product. For reactions with a neutral transition state, for instance the prominent DielsAlder reaction, this is a very challenging task since product and transition state are structurally closely related. Chemists are not yet able to predictably tackle such design problems. For reactions with charged transition states but neutral products the problem of product inhibition is obviously less severe.

\subsection{Potential Advantages of Catalysis inside Supramolecular Containers}

Why is the exploration of supramolecular containers as catalytic host structures worthwhile? For a detailed discussion, we refer the interested reader to our recent concept article ${ }^{22]}$ on this topic, and only summarize the advantages here briefly. Generally, there are three main advantages when performing reactions inside containers: 1) Product selectivity: due to the inter- actions of the substrate with the host, a different reaction pathway may become available, leading to a different product than in the regular solution phase. 2) Substrate selectivity: due to the confined environment inside the host structure, only substrates that fit are converted. Although this can be regarded as a disadvantage at first, it may be of advantage for the selective conversion of complex substrate mixtures in the growing field of systems chemistry.[23] Additionally, it also contributes to the third advantage: 3) Multicatalyst tandem reactions. Performing several consecutive reactions in solution, without work-up after each step, is a daunting challenge. All the different reagents and catalysts may react with each other and not with the substrate in the desired order. Supramolecular containers may alleviate these problems by segregating the catalytically active centers and providing a high substrate selectivity. ${ }^{[24,25]}$

\section{Examples from our Lab}

In our group, we started by exploring known supramolecular containers for catalytic applications, since a large variety of structures is known but very few have been explored for their potential use in catalysis. We were especially interested in hexamer I, which was first reported by the group of Atwood.[9] It self-assembles from six resorcin[4]arene units $\mathbf{1}$ (Fig. 1) and eight water molecules in apolar solvents and encloses a cavity of approx. $1.4 \mathrm{~nm}^{3}$. It was shown that the system is able to reversibly encapsulate a variety of guests and that it displays a high affinity for cationic species like tetraalkyl ammoniums due to cation- $\pi$ interactions. ${ }^{[26-28]}$ Interestingly, it was also reported that neutral tertiary amines are encapsulated well inside the system, ${ }^{[29]}$ and that seemed intriguing to us since there should be no strong interactions between amines and the aromatic cavity. We reinvestigated this issue and found that amines are only encapsulated well because they are protonated by the capsule $\mathbf{I}$, forming a cationic ammonium species which then benefits from cation- $\pi$ and coulombic interactions on the inside of I (Scheme 1). ${ }^{[30]}$

We determined the acidity of the hexamer ( $\mathrm{p} K_{\mathrm{a}}$ approx. 5.5-6), which is about four orders of magnitude more acidic than a regular phenol. Stabilization of the nega-

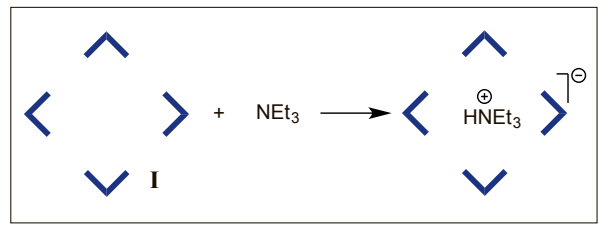

Scheme 1. Amines are encapsulated as pronated species inside $\mathbf{I}$.

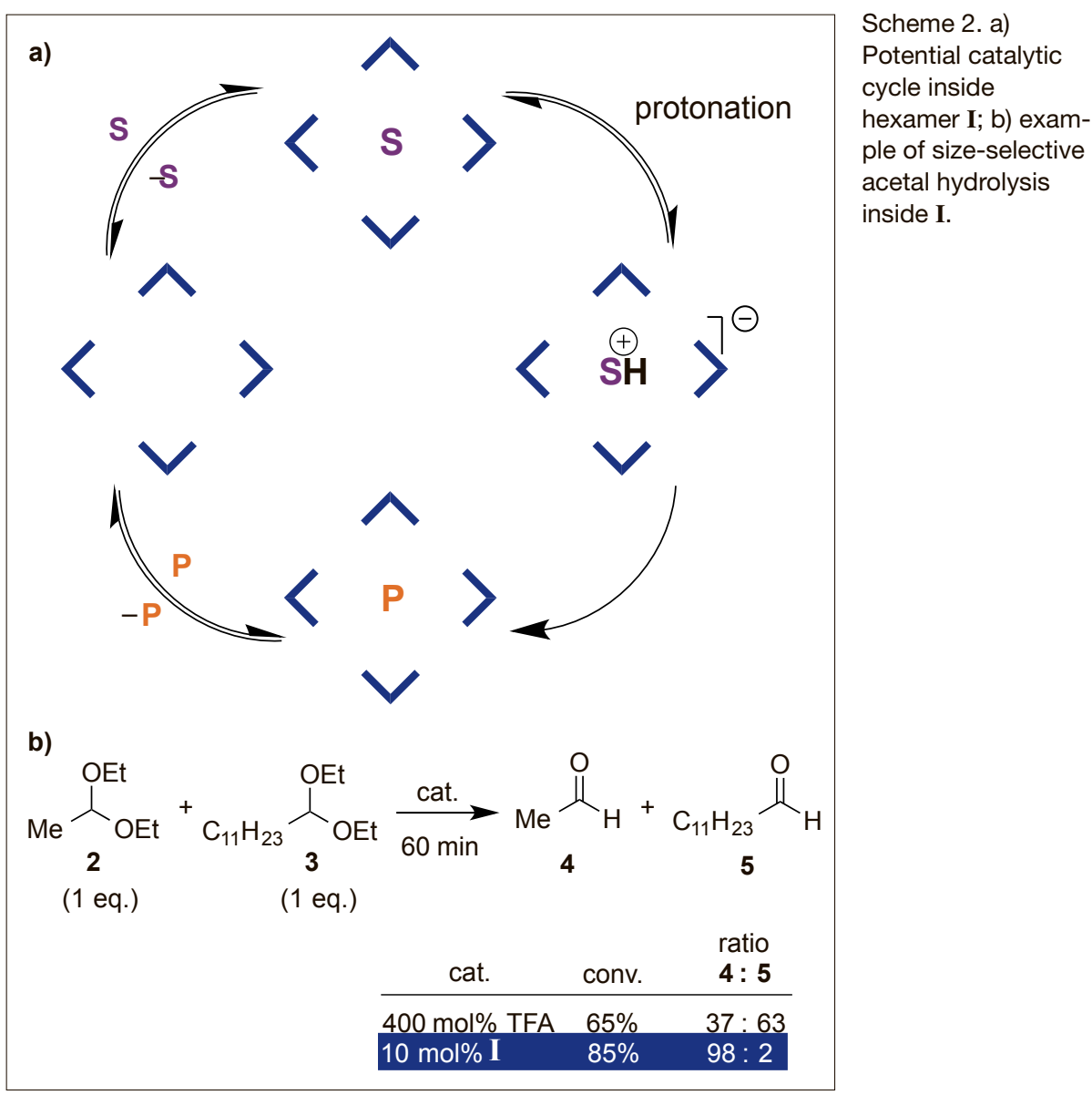


tive charge throughout the hydrogen bond network, as well as cation- $\pi$ and coulombic interactions with the protonated amine are the likely cause for the acidity increase. The fact that such an important property of I was revealed by us more than fifteen years after its discovery, may hint at the underexplored potential of known supramolecular structures. Why was the discovery of the acidity of I so important for catalytic applications? It provided us with an assembly that may activate suitable substrates by protonation and then stabilizes cationic transition states within its cavity due to coulombic and cation- $\pi$ interactions. A potential catalytic cycle is displayed in Scheme 2a. The uptake of suitably sized substrates should not pose a challenge, since guest uptake in this system is well established. ${ }^{[28]}$ The acidity of I would then allow the activation of a suitable guest by protonation. For a rate acceleration inside the hexamer, as compared to the reaction outside in bulk solution, cationic transition states would have to be stabilized to a greater extent than cationic intermediates, as discussed before. After the completion of the reaction, the product has to be released from the pocket, to complete a catalytic cycle. If the product is not cationic in nature, this should not pose a challenge since there will be no strong interactions with the interior.

As a first test reaction, we explored simple hydrolysis of diethyl acetals. Indeed, we observed a rate acceleration in the presence of $\mathbf{I}^{\left[{ }^{[30]} \text { What kind of evidence }\right.}$ do we have that the reaction indeed takes place inside the cavity and not outside in solution or on the outer surface? First, if we block the cavity space with a strong binding cationic guest (tetrabutyl ammonium bromide), no acceleration is detected. Even more convincing is a competition experiment between two differently sized acetals. If the reaction takes place inside the hexamer, then the substrate which enters the cavity more efficiently should be hydrolyzed selectively in the presence of the larger acetal. That is exactly what we observed: The small 1,1-diethoxyethane (2) was hydrolyzed in the presence of 1,1-diethoxydodecane (3) in excellent selectivity (98:2) with $10 \mathrm{~mol} \%$ of I (Scheme 2b). In solution, using a regular Brønsted acid like for instance trifluoroacetic acid (TFA), such a selectivity cannot be achieved. This example, therefore, nicely demonstrates the concept of substrate selectivity.

\subsection{Terpene Cyclization}

After having demonstrated that capsule I is indeed suited for catalyzing reactions with cationic transition states, we wanted to further explore its potential. We were and still are especially interested in the "tail-to- head' [31] terpene (THT) cyclization, which in contrast to the 'head-to-tail' version is very hard to control in solution. Nature has evolved highly specialized enzymes for this task, and man-made catalysts able to perform selective THT-cyclizations are lacking. In nature, the pyrophosphate leaving group is activated inside the enzyme with a magnesium(II)-Lewis acid and cationic intermediates and transition states are stabilized by cation- $\pi$ interactions. ${ }^{[20,21]}$ Depending on the conformation of the flexible acyclic terpene in the enzyme pocket, different (poly)cyclic structures are formed by complex cationic cascade reactions (for a few examples see Scheme $3 a)$. We speculated that we may be able to mimic the basic mode of operation of such enzymes with the much simpler hexamer I, which can function as a Brønsted acid and is able to stabilize cationic species.

Indeed, under optimized conditions we were able to perform THT-cyclizations under very mild conditions: $30^{\circ} \mathrm{C}, 10 \mathrm{~mol} \%$ of I in chloroform solution. ${ }^{[32]}$ The commercially available nerol (6), for instance, was cyclized to the bicyclic eucalyptol (8) in relatively good yield $(40 \%$, Scheme $3 b)$. Such a direct conversion has not been described before utilizing man-made catalysts. Especially interesting was the con- version of geranyl acetate (9) to mainly $\alpha$-terpinene (10, Scheme 3c). There were no intermediate products observed during the reaction, although they can be easily detected since in contrast to natural enzymes they do not bind strongly to the pocket. This indicates that a direct isomerization of the transoid allylic cation $\mathbf{1 1}$ to the cisoid form $\mathbf{1 2}$ has to occur. Such a direct isomerization was excluded in the proposed biosynthesis ${ }^{[33]}$ due to gas phase calculations which indicated a relatively high energy barrier ( $c a .55 \mathrm{~kJ} / \mathrm{mol}$ ) for this process. ${ }^{[34]}$ Our results suggest that such isomerizations are feasible inside artificial catalyst systems and therefore we believe they should not be categorically excluded for the proposed biosynthesis any longer. Work to further clarify this issue is ongoing.

\subsection{Hydroalkoxylation}

Inspired by the efficient conversion of the intermediary alcohol 7 to the cyclic ether eucalyptol (8, Scheme 3b), we further investigated the scope of the hexamer I-catalyzed intramolecular hydroalkoxylation of unactivated hydroxy olefins. ${ }^{[35]}$ Applying $10 \mathrm{~mol} \%$ of catalyst $\mathbf{I}$ at $30{ }^{\circ} \mathrm{C}$, several tetrahydropyran $($ e.g. 14, 15, 16) and oxepane $($ e.g. 17, 18) derivatives could

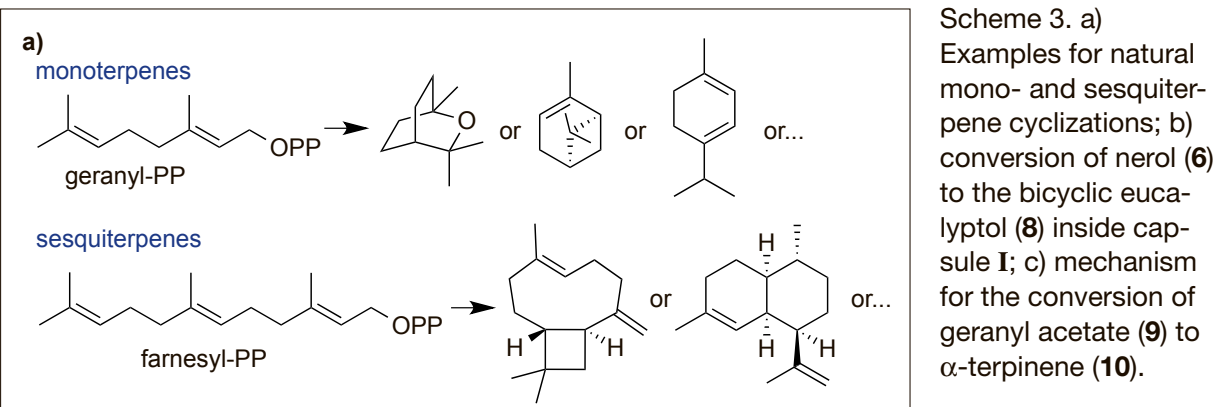

b)<smiles>CC(C)=CCC/C(C)=C\CO</smiles>

c)

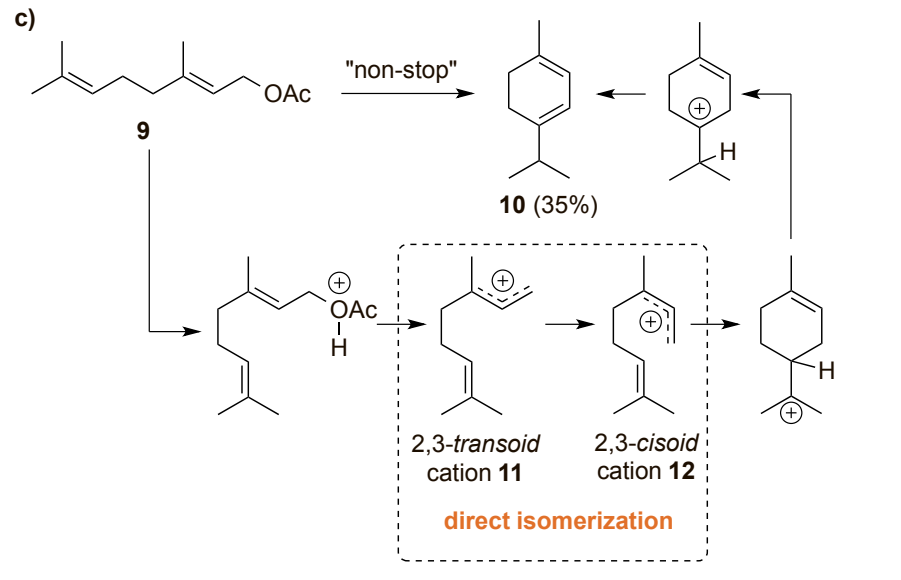


be successfully synthesized in generally good to excellent yields (see Scheme 4a for a few examples). Additionally, all substrates tested only showed low conversions in the presence of a competitive inhibitor (tetrabutyl ammonium bromide), indicating that the reactions proceed within the capsule interior. Further evidence was provided by the selective conversion (92:8) of the small substrate $\mathbf{1 3}$ in the presence of the long chain analogue 19 (Scheme 4b). The mild reaction conditions employed contrast the often harsh conditions required when utilizing regular Brønsted acids in solution. This study therefore further corroborates the applicability of supramolecular catalysts to standard organic transformations.

\subsection{Iminium Catalysis}

Beside intramolecular reactions, we are also interested in intermolecular conversions which, as discussed before, often suffer from severe product inhibition. We therefore focused on reactions where the product does not show strong interactions with the cavity interior. The iminiumcatalyzed 1,4-addition of nucleophiles to $\alpha, \beta$-unsaturated aldehydes seemed ideal for this purpose: the reactive electrophile is formed by the condensation of a secondary amine (for instance L-proline) with the $\alpha, \beta$-unsaturated aldehyde. This reactive species should be an excellent guest for capsule I as long as its size fits the cavity dimensions. If the iminium species is encapsulated fast enough, the reaction would have to occur inside the limited space of I (Scheme 5a), where different selectivities than in bulk solution should be observable. We found that the 1,4-reduction with Hantzsch ester 22 (Scheme 5b) is a suitable reaction for this purpose. [36] With L-proline as catalyst, we found that the yield of aldehyde 23a as well as the enantiomeric excess obtained are much higher $(74 \% e e)$ in the presence of capsule $\mathbf{I}$ as compared to otherwise identical bulk solution conditions $(9 \% e e)$, resulting in a $\Delta e e$ of $65 \% .{ }^{[37]}$ The substrate scope was investigated and, as expected for a reaction inside a densely packed environment, a high dependence on the substitution pattern was observed (Scheme 5c). Especially ortho-substituted phenyl derivatives displayed a pronounced selectivity in the presence of container I (Scheme 5b). A $\Delta e e$ of $92 \%$ was observed in the case of the ortho-methyl derivative 21d. What is causing the modulation of enantiomeric excess inside the cavity? A first indication was obtained with cyclohexylderivative 21f. Its reduced $\Delta e e$ indicates that a phenyl group might be essential to obtain a high modulation. We propose that the iminium species binds to the inner walls of the cavity from the less hindered side (anti to the carboxylic acid in the case of

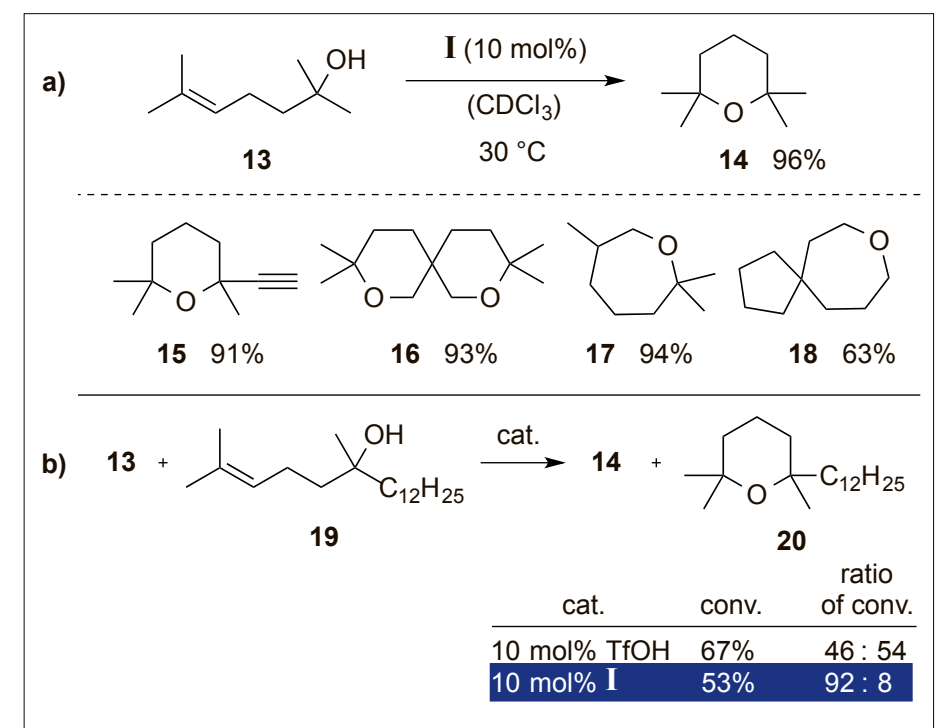

Scheme 4. a) Intramolecular hydroalkoxylation catalyzed by hexamer I; b) size-selectivity in the conversion to the cyclic ethers 14 and 20.
L-proline, Scheme 5d). This binding favors the attack of the nucleophile from the top face, delivering more of the $S$-product than in the regular solution experiment. These results indicate that the non-covalent combination of supramolecular systems with iminium catalysis might be helpful in improving enantioselectivity for challenging reactions.

\section{Outlook}

Our first results clearly indicate the potential of catalysis inside supramolecular containers. Nevertheless, our results, as well as the results from the Scarso and Strukul groups with catalyst $\mathbf{I},{ }^{[38-41]}$ represent only proof-of-principle studies and several challenges will have to be tackled before such methods become suitable for general applications. So far it is not possible to predictably design novel catalytically active host structures. Especially for terpene cyclizations, where a multitude of different complex polycyclic ring systems can be formed, the rational design of the cavity space would be required to access cyclization products in a selective fashion. Not too surprisingly, most supramolecular hosts available are of very high symmetry since they are formed by the self-assembly of multiple copies of one building block. Novel ways to access less symmetric and therefore more selective cavity shapes will have to be developed.

\section{Summary}

Herein we have summarized our first results concerning catalysis inside the known resorcin[4]arene host $\mathbf{I}$. These results rely on our findings that the host is surprisingly acidic and therefore able to activate suitable substrates by protonation. We have collected evidence that the reactions described indeed occur inside the supramolecular system. Inside the host, cationic species are stabilized by cation- $\pi$

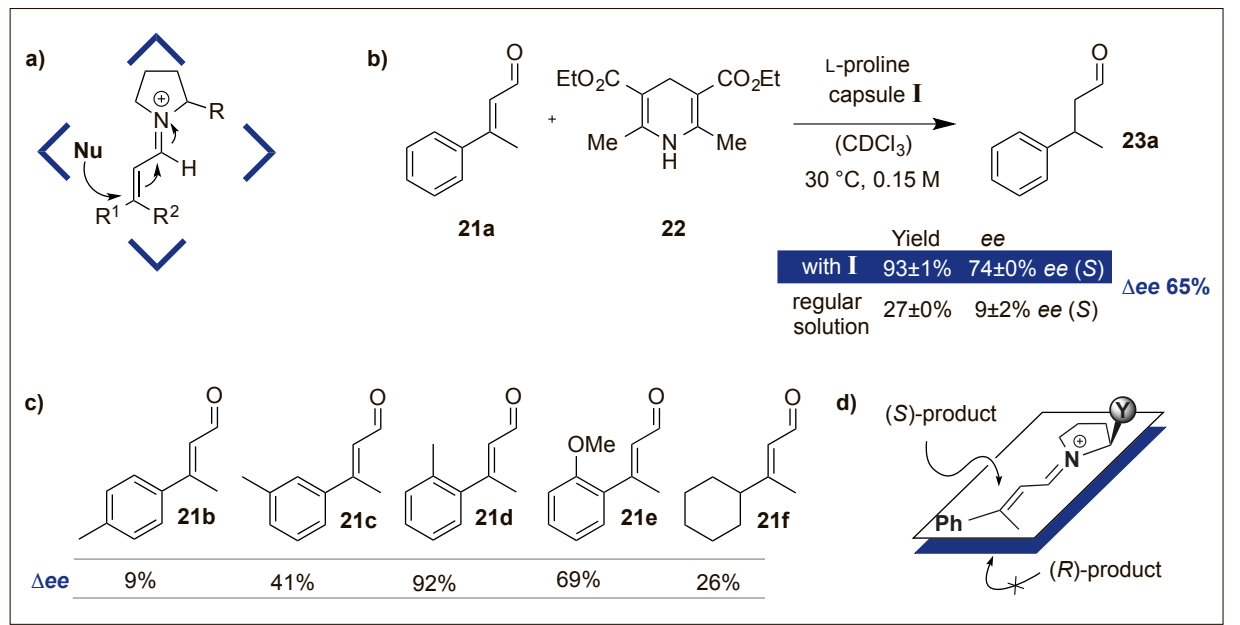

Scheme 5. a) Reaction of the entrapped iminium species; b) iminium catalyzed reduction investigated in the presence and absence of capsule $\mathbf{I}$; c) substrate scope of the reaction; hypothesis for the observed modulation of the enantiomeric excess. 
and also coulombic interactions. These interactions can lead to an acceleration of reactions by stabilizing cationic transition states. Additionally, the limited volume inside the host can lead to novel or improved selectivities as compared to the results in the regular bulk solvent. Further investigations are ongoing to elucidate the full potential of this host as well as other supramolecular structures.

\section{Acknowledgements}

Support was provided by the Bayerische Akademie der Wissenschaften and the Fonds der Chemischen Industrie. T. M. B. thanks the National Research Fund, Luxembourg for an AFR fellowship.

Received: September 2, 2016

[1] A. J. Kirby, Angew. Chem. Int. Ed. 1996, 35, 707.

[2] R. Warmuth, in 'Supramolecular Chemistry', John Wiley \& Sons, Ltd, Chichester, UK, 2012, DOI: 10.1002/9780470661345.smc054.

[3] M. Yoshizawa, M. Fujita, Bull. Chem. Soc. Jpn. 2010, 83, 609 .

[4] T. K. Ronson, S. Zarra, S. P. Black, J. R Nitschke, Chem. Commun. 2013, 49, 2476.

[5] M. Han, D. M. Engelhard, G. H. Clever, Chem. Soc. Rev. 2014, 43, 1848.

[6] G. Zhang, M. Mastalerz, Chem. Soc. Rev. 2014, $43,1934$.
[7] D. Ajami, L. Liu, J. Rebek Jr, Chem. Soc. Rev. 2015, 44, 490 .

[8] J. H. Jordan, B. C. Gibb, Chem. Soc. Rev. 2014, 44, 547.

[9] L. R. MacGillivray, J. L. Atwood, Nature 1997, 389, 469.

[10] D. M. Vriezema, M. Comellas Aragonès, J. A A. W. Elemans, J. J. L. M. Cornelissen, A. E. Rowan, R. J. M. Nolte, Chem. Rev. 2005, 105, 1445.

[11] T. S. Koblenz, J. Wassenaar, J. N. H. Reek, Chem. Soc. Rev. 2008, 37, 247.

[12] M. D. Pluth, R. G. Bergman, K. N. Raymond, Acc. Chem. Res. 2009, 42, 1650.

[13] M. Yoshizawa, J. K. Klosterman, M. Fujita, Angew. Chem. Int. Ed. 2009, 48, 3418.

[14] M. J. Wiester, P. A. Ulmann, C. A. Mirkin, Angew. Chem. Int. Ed. 2011, 50, 114.

[15] C. J. Brown, F. D. Toste, R. G. Bergman, K. N. Raymond, Chem. Rev. 2015, 115, 3012.

[16] S. H. A. M. Leenders, R. Gramage-Doria, B. de Bruin, J. N. H. Reek, Chem. Soc. Rev. 2015, 44, 433.

[17] L. Catti, Q. Zhang, K. Tiefenbacher, Synthesis 2016, 48, 313.

[18] P. Mal, B. Breiner, K. Rissanen, J. R. Nitschke, Science 2009, 324, 1697.

[19] M. D. Pluth, R. G. Bergman, K. N. Raymond, Science 2007, 316, 85 .

[20] C. A. Lesburg, G. Zhai, D. E. Cane, D. W. Christianson, Science 1997, 277, 1820 .

[21] C. M. Starks, K. Back, J. Chappell, J. P. Noel, Science 1997, 277, 1815.

[22] L. Catti, Q. Zhang, K. Tiefenbacher, Chem. Eur. J. 2016, 22, 9060 .

[23] E. Mattia, S. Otto, Nat. Nanotechnol. 2015, 10 111
[24] Z. J. Wang, K. N. Clary, R. G. Bergman, K. N. Raymond, F. D. Toste, Nat. Chem. 2013, 5, 100

[25] A. G. Salles, S. Zarra, R. M. Turner, J. R. Nitschke, J. Am. Chem. Soc. 2013, 135, 19143.

[26] A. Shivanyuk, J. Rebek, Proc. Natl. Acad. Sci. USA 2001, 98, 7662.

[27] L. Avram, Y. Cohen, J. Am. Chem. Soc. 2002, 124, 15148.

[28] L. Avram, Y. Cohen, J. Rebek, Chem. Commun. 2011, 47, 5368

[29] L. Avram, Y. Cohen, J. Am. Chem. Soc. 2003, $125,16180$.

[30] Q. Zhang, K. Tiefenbacher, J. Am. Chem. Soc. 2013, 135, 16213.

[31] S. V Pronin, R. A. Shenvi, Nat. Chem. 2012, 4, 915.

[32] Q. Zhang, K. Tiefenbacher, Nat. Chem. 2015, 7, 197.

[33] R. Croteau, Chem. Rev. 1987, 87, 929

[34] N. L. Allinger, J. H. Siefert, J. Am. Chem. Soc. 1975, 97, 752.

[35] L. Catti, K. Tiefenbacher, Chem. Commun. 2015, 51, 892 .

[36] J. W. Yang, M. T. Hechavarria Fonseca, B. List, Angew. Chemie Int. Ed. 2004, 43, 6660.

[37] T. M. Bräuer, Q. Zhang, K. Tiefenbacher, Angew. Chem. Int. Ed. 2016, 55, 7698.

[38] G. Bianchini, G. La Sorella, N. Canever, A. Scarso, G. Strukul, Chem. Commun. 2013, 49 , 5322.

[39] S. Giust, G. La Sorella, L. Sperni, F. Fabris, G. Strukul, A. Scarso, Asian J. Org. Chem. 2015, 4, 217.

[40] G. La Sorella, L. Sperni, G. Strukul, A. Scarso, Chem CatChem 2015, 7, 291.

[41] G. La Sorella, L. Sperni, P. Ballester, G. Strukul, A. Scarso, Catal. Sci. Technol. 2016, 6, 6031. 